Abanico Veterinario. Enero-Diciembre 2020; 10:1-8. http://dx.doi.org/10.21929/abavet2020.19

Nota Corta. Recibido: 02/03/2020. Aceptado: 10/07/2020. Publicado: 28/07/2020.

\title{
Edad de sacrificio y tiempo de muestreo sobre pH y color de la carne de ovinos de pelo
}

\author{
Sampling time and age at sacrifice over $\mathrm{pH}$ and meat color in hair sheep \\ Jaramillo-López Esaúl* ID, Peraza-Mercado Gwendolyne ID, Itzá-Ortiz Mateo ID
}

Universidad Autónoma de Ciudad Juárez, Instituto de Ciencias Biomédicas, Avenida Plutarco Elías Calles, \# 1210, Fovissste Chamizal, CP. 32310, Ciudad Juárez, Chihuahua, México. *Autor responsable y de correspondencia: Jaramillo-López Esaúl, Departamento de Ciencias Veterinarias, del Instituto de Ciencias Biomédicas de la Universidad Autónoma de Ciudad Juárez. Ciudad Juárez, Chihuahua, México. ejaramil@uacj.mx,gperaza@uacj.mx,mateo.itza@uacj.mx.

RESUMEN

El objetivo del presente trabajo fue evaluar la edad al sacrificio, hora de muestreo de la canal y músculo sobre el pH y color de la carne en corderos y ovejas. Se utilizaron 25 ovinos, 14 hembras y 11 machos, con un peso de $41.06 \pm 0.064 \mathrm{~kg}$ y $28.244 \pm 2.435$, respectivamente. La edad de las hembras fue de 2.32 años y los machos menores de 1 año, de fenotipos de pelo indefinidos. Se sacrificaron por el método del degüelle. Después de la evisceración se realizó la primera lectura de $\mathrm{pH}$, así como el color de la carne, posteriormente se tomó una muestra del músculo Femoral, 24 h después se realizó la segunda lectura del $\mathrm{pH}$ y color en este músculo. Los datos obtenidos se analizaron con un diseño de bloques completamente al azar. El tiempo de muestreo afecto el pH $(\mathrm{P}<0.01)$ al tiempo cero fue de $6.081 \pm 0.057$ vs $5.264 \pm 0.017$. Para el color no hubo efecto $(P>0.05)$ de la edad para, $L^{*} a^{*} y b^{*}$. Sin embargo, el tiempo de muestreo afecto los valores de $L^{*}$ y $b^{*}(P<0.01)$. La edad no afecto el $\mathrm{pH}$ y el color de la carne, sin embargo, la hora de muestreo afecto ambas variables.

Palabras claves: tiempo de muestreo, color, $\mathrm{pH}$.

\begin{abstract}
The objective of the present work was to evaluate the age at slaughter, carcass and muscle sampling time on the $\mathrm{pH}$ and meat color in lambs and sheep. The 25 sheep, 14 females and 11 males, weighing $41.06 \pm$ $0.064 \mathrm{~kg}$ and $28.244 \pm 2.435$, respectively, were used. The age of the females was 2.32 years, and the males younger than 1 year, of undefined hair phenotypes. They sacrificed themselves by the disgorging method. After evisceration, the first $\mathrm{pH}$ reading was taken, as well as the color of the meat, then a sample of the Femoral muscle was taken, $24 \mathrm{~h}$ later, the second $\mathrm{pH}$ and color reading was taken in this muscle. The data obtained was analyzed with a completely randomized block design. The sampling time affected the $\mathrm{pH}(\mathrm{P}<0.01)$ at zero time was $6,081 \pm 0.057$ vs $5,264 \pm 0.017$. For color there was no effect $(P>0.05)$ of age for, $L^{*}, a^{*}$ and $b^{*}$. However, the sampling time affected the $L^{*}$ and $b$ * values $(P<0.01)$. Age did not affect the $\mathrm{pH}$ and color of the meat, however, the sampling time affected both variables.
\end{abstract}

Keywords: time-sampling, color, $\mathrm{pH}$. 


\section{INTRODUCCIÓN}

La producción en México de ganado ovino en 2016 fue de aproximadamente 118 mil toneladas, de las que se destinaron para carne en canal; 60,300 ton. El $95 \%$ de la carne de borrego en México se consume en forma de barbacoa y sólo el $5 \%$ se consume de otra manera; en la que se incluyen los cortes finos. La producción ovina sólo cubre el $70 \%$ de la demanda nacional, el 30\% restante es importada de Australia, Nueva Zelanda y USA (SAGARPA, 2017).

El color de la carne es determinante para elegir o rechazar al momento de la compra por parte de los consumidores. Las preferencias del consumidor por un determinado aspecto del color del músculo, carnes rosadas o rojas, varían en función del tipo de consumidor; así como de la costumbre del mercado local. El color de la carne se debe a la concentración de pigmentos (mioglobina), su estado químico y las propiedades de la dispersión de la luz en la carne (Alberti y Ripoll, 2010; Calnan et al., 2014).

El color de la carne fresca de rumiantes está determinado por factores endógenos y exógenos; así como la complejidad de las interacciones entre estos (AMS, 2012; Neethling et al., 2017). Dentro de los factores endógenos se encuentran la especie, raza, edad al sacrificio y el músculo en donde se toman las muestras. Entre los factores exógenos más importantes, es el sistema de alimentación (extensivo vs intensivo) Rodrigues et al. (2011), y el estrés ante morten Neethling et al. (2017).

El color de la carne se afecta por la nutrición, velocidad de enfriamiento de la canal, músculo, localización de la muestra dentro del músculo, pH del músculo, temperatura, tiempo de almacenamiento post morten, tiempo de exposición al oxígeno y concentración de mioglobina (AMS, 2012; Neethling et al., 2017).

Estudios realizados por Pascual-Alonso et al. (2015), quienes evaluaron el color en corderos lechales y ternascos, sacrificados a un peso de $10.97 \pm 0.3 \mathrm{~kg}$ y 30 días de edad y corderos ternascos, sacrificados a un peso de $16 \pm 0.72 \mathrm{~kg}$ y 60 días de edad; el color de la carne fue más clara con menos pigmentos en los corderos lechales, con valores de luminosidad $\left(\mathrm{L}^{*}\right) 47.82 \pm 0.98$ vs $43.6 \pm 0.9$ y para la rojeza $\left(\mathrm{a}^{*}\right)$ de $13.91 \pm 0.53$ vs $15.35 \pm 0.53$, respectivamente. Guerrero et al. (2013), evaluaron el peso y edad de corderos ternascos y corderos al sacrificio, con relación al color de la carne, 21.9 vs 26.5 kg y 93 y 133 días, encontraron que la carne de cordero es más oscura.

Lo anterior lleva a la conclusión que en corderos conforme aumenta el peso al sacrificio, el color rojo es más intenso (Marichal et al., 2003; žgur et al., 2003; Calnan et al., 2014). Por otra parte, se ha reportado un efecto del genotipo del cordero y del cabrito sobre el color de la carne (Rodrigues et al., 2011; Fernandes et al., 2013). 
Los animales alimentados en sistemas extensivos realizan una mayor actividad física, en comparación a los animales alimentados en sistemas intensivos; lo cual resulta que la carne de sistemas extensivos contenga un menor número de fibras contráctiles, un alto potencial metabólico oxidativo; lo cual hace aumentar la proporción de fibras rojas oxidativas, por lo que la carne tiene una apariencia más oscura que los que se crían en sistemas intensivos (Alberti y Ripoll, 2010; Rodrigues et al., 2011; Neethling et al., 2017). La adición de zilpaterol en la dieta de ovinos disminuye el índice de rojeza ( $a^{\star}$ ) en la carne (Partida et al., 2015).

El color de la carne de ovinos en México, se ha determinado principalmente en corderos; sin embargo, en el estado de Chihuahua no hay información disponible para las hembras adultas, por lo que es importante generar información al respecto.

Otra de las características físicas importantes de la calidad que afectan la carne de los ovinos es el $\mathrm{pH}$, es debido a que se ha demostrado que afecta la vida de anaquel, la terneza y el color de la carne; el valor ideal debe ser entre un pH de 5.5 a 5.8 (Taruman, et al., 2018). El pH se ve afectado por la raza de ovino (Young et al., 1993), en cabritos también se han reportado efectos del fenotipo (Rodrigues et al., 2011), pero no se afecta por el sexo (Craigie et al., 2012) ni por el tiempo de alimentación (Rezende et al., 2017). El pH se afecta, por el estrés antemorten. Se ha demostrado una relación entre el tiempo de transporte y el número de hematomas en corderos, al medir el $\mathrm{pH}$ en el músculo Longissimus Thoracis fue de 5.8 a 6.3, y al medirse en la pierna en los músculos Semitendinosus y Biceps femoris, fue más más estable $\mathrm{pH}<5.8$ (Taruman et al., 2018). En México hay poca información sobre el pH y el color de la carne de ovinos de pelo. En el estado de Chihuahua la información es aún menor y se carece de información para las ovejas.

El objetivo del presente trabajo fue evaluar la edad al sacrificio, hora de muestreo y el sexo; sobre el $\mathrm{pH}$ y color de la carne en ovinos de pelo.

\section{MATERIAL Y MÉTODOS}

El trabajo se realizó en el área de Alimentación de Rumiantes, del Departamento de Ciencias Veterinarias del Instituto de Ciencias Biomédicas, de la Universidad Autónoma de Ciudad Juárez, del estado de Chihuahua, México. El experimento se realizó durante el periodo del 3 de septiembre al 12 de noviembre de 2017; se utilizaron 25 ovinos, 14 hembras de una edad de 2.32 años, y con un peso de $41.064 \pm 1.744 \mathrm{~kg}$ y 11 machos menores de un año de edad, y con un peso de $28.244 \pm 2.435 \mathrm{~kg}$.

El fenotipo fue indefinido, ya que había animales en los que predominaba la raza Dorper, en otros Pelibuey, Panza Negra, Rambouillet y las cruzas entre estas razas; así como ovinos criollos. Los animales se alimentaron con alfalfa henificada y pastos nativos. Los 
ovinos se compraron el día del sacrificio en las granjas de la periferia de Ciudad Juárez, a las 6:00 h.

Los ovinos fueron pesados antes del sacrificio, usando una báscula digital marca Detecto, modelo CN 20; se sacrificaron a las 8:00 de la mañana por medio del método del degüelle, con base a la norma oficial mexicana (NOM-033-ZOO-1995), sobre sacrificio humanitario de los animales, posterior a la evisceración y desuelle de los animales. Se procedió a determinar el $\mathrm{pH}$ y color de la carne, en el tiempo 0; después se tomó una muestra del músculo Femoral, se identificó, se guardó en refrigeración a $2{ }^{\circ} \mathrm{C}$, por $24 \mathrm{~h}$, para realizar la segunda determinación de color y pH. Se tomó la muestra del músculo Femoral, porque es más estable el pH, en este músculo (Taruman et al., 2018)

El pH de la carne se determinó después de la evisceración (tiempo 0), en el músculo Femoral; se utilizó un potenciómetro de penetración en carne (Hanna HI 99163), que fue calibrado con buffer de 7.0. Después se tomó una muestra del músculo Femoral, se guardó en refrigeración a $2^{\circ} \mathrm{C}$. La segunda lectura se realizó a las $24 \mathrm{~h}$, después de que se tomó la muestra. Cada una de las lecturas se realizó por triplicado.

Para el color se utilizó un colorímetro (Konica Minolta CR 400), con el cual se evaluó la claridad $L^{*}$, los valores positivos de $a^{*}$ corresponden al rojo y los valores positivos de $b^{*}$ corresponden al amarillo (Alberti y Ripoll, 2010). Se hicieron dos lecturas del músculo Femoral, al tiempo 0 y a las $24 \mathrm{~h}$. Cada una de las lecturas se realizó con tres repeticiones. Los datos obtenidos se analizaron con un diseño por bloques completamente al azar, en donde las variables independientes fueron: el tiempo de lectura (0 y $24 \mathrm{~h}$ ), edad al sacrificio (2.32 años y menores a 1 año) y el sexo (hembras y machos); las variables dependientes fueron color y $\mathrm{pH}$ de la carne. Se analizó por el uso del paquete estadístico SPSS versión 25 para Windows, 2017.

\section{RESULTADOS Y DISCUSIÓN}

Los resultados obtenidos se presentan en la tabla 1, en donde se muestra el valor medio y el error estándar, para la edad, hora de muestreo y sexo. No se encontró efecto de la edad y sexo sobre el $\mathrm{pH}$ y color $(\mathrm{P}>0.05)$; pero la hora de muestreo fue altamente significativa $(P<0.01)$, valores mayores de $L^{*} y b^{*}$ se registraron a las $24 h$; sin embargo, el $\mathrm{pH}$ fue menor a las $24 \mathrm{~h}$ post morten.

Alberti y Ripoll, (2010) reportaron valores de $L^{*} 48.3$, para $a^{*} 10.3$ y b*11.2, que son completamente diferentes a los encontrados en este trabajo; estas diferencias pueden deberse al sistema de alimentación, ya que los animales engordados en pastos tienden a producir carne con menos luminosidad, debido a un aumento en el ejercicio, lo que favorece que aumente la proporción de fibras rojas oxidativas (Rodríguez et al., 2011; Neething et al., 2017). No se encontró efecto de la edad sobre el color de la carne, estos 
resultados difieren a los reportado por Guerrero et al., (2013), quienes sacrificaron corderos a los 93 y 133 días; encontraron que a mayor edad al sacrificio, la carne es más oscura. Esta diferencia puede deberse a que en el presente trabajo los corderos fueron de un año de edad y las ovejas de 2.32.

Tabla 1. Color y pH de la carne en ovinos de pelo

\begin{tabular}{|c|c|c|c|c|}
\hline & \multicolumn{2}{|c|}{ Color } & & $\mathrm{pH}$ \\
\hline \multicolumn{5}{|l|}{$\begin{array}{l}\text { Edad al } \\
\text { sacrificio }\end{array}$} \\
\hline 2.32 años & $36.095 \pm 0.477$ & $16.082 \pm 0.299$ & $6.853 \pm 0.349$ & $5.74 \pm 0.075$ \\
\hline \multirow[t]{2}{*}{$<1$ año } & $35.323 \pm 0.474$ & $15.378 \pm 0.294$ & $7.544 \pm 0.270$ & $5.629 \pm 0.057$ \\
\hline & ns & ns & ns & ns \\
\hline Hora de & muestreo & & & \\
\hline 0 horas & $34.279^{a} \pm 0.352$ & $16.23 \pm 0.270 \mathrm{~ns}$ & $5.724^{\mathrm{a}} \pm 0.250$ & $6.081^{b} \pm 0.057^{* \star}$ \\
\hline \multirow[t]{2}{*}{24 horas } & $36.971^{\mathrm{b}} \pm 0.352^{* *}$ & $15.069 \pm 0.310$ & $8.823^{b} \pm 0.243^{\star *}$ & $5.264^{a} \pm 0.017$ \\
\hline & ** & ns & ** & ** \\
\hline \multicolumn{5}{|l|}{ Sexo } \\
\hline Hembras & $35.323 \pm 0.474$ & $15.37 \pm 0.294$ & $7.54 \pm 0.270$ & $5.629 \pm 0.057$ \\
\hline \multirow[t]{2}{*}{ Machos } & $36.095 \pm 0.477$ & $16.02 \pm 0.290$ & $6.53 \pm 0.394$ & $5.74 \pm 0.075$ \\
\hline & ns & ns & ns & ns \\
\hline
\end{tabular}

El efecto de la hora de lectura del color de la carne, sí tuvo diferencias $(P<0.01)$. Los valores de $L^{*}$ y $b^{*}$ a la hora del sacrificio y medido a las $24 \mathrm{~h}$ después, fueron de $34.28 \mathrm{y}$ $36.97 ; 5.72$ y 8.82 respectivamente; para $a^{*}$ no hubo diferencias $(P>0.05)$. La diferencia puede deberse al proceso de maduración de la carne; así como al descenso del pH (Taruman, et al., 2018). Alberti y Ripoll, (2010), recomiendan que la medición del color de la carne debe de iniciarse a las $24 \mathrm{~h}$, del sacrificio.

El sexo no tuvo diferencias $(P>0.05)$ sobre el color de la carne, el valor de $L^{*}$ fue de 35.32 y 36.07, para hembras y machos; sin embargo, Žgur et al. (2003) reportaron valores de $L^{*}$ de 39.02 y 41.48 , para hembras y machos; esta diferencia la atribuyen al crecimiento de los machos, ya que crecen más rápido que las hembras.

Es importante mencionar que en el presente trabajo las hembras tuvieron mayor edad que los machos, y lo observado por žgur et al. (2003), pudiera no haberse demostrado. Por otra parte Bianchi (2006), no encontró efecto del sexo al analizar el color de la carne de corderos; los valores de $L^{*}, a^{*}$ y $b^{*}$ para hembras y machos fueron: 40.4 y 39.8; 17.6 y 17.4 y 10.3 y 10.3. Estos valores difieren a los encontrados en el presente trabajo que fueron de: 35.32 y $36.06 ; 15.37$ y $16.06 ; 7.54$ y 6.53 respectivamente para $L^{*}, a^{*}$ y b*. Las diferencias pueden deberse al control de la alimentación; ya que en el presente trabajo no se controló; los animales se compraron en granjas de la periferia de la ciudad. 
La edad al sacrificio no afectó el pH de la carne. Estos resultados son similares a los reportados por Rezende et al. (2017), quienes reportaron valores de pH de 5.7 y 5.5, para corderos sacrificados a 140 y 182 días.

El pH medido a las 0 y $24 \mathrm{~h}$ fue de 6.081 y 5.264 , que fue similar a lo reportado por Rezende et al. (2017), quienes reportaron valores de pH entre 6.0 y 5.7. El descenso del $\mathrm{pH}$ se debe a la glucólisis anaeróbica en el músculo, lo que aumenta la vida de anaquel y mejora las características organolépticas de la carne, Craigie et al. (2012) reportaron valores de pH de 5.58. Para el pH de la carne McGeehin (2001) no encontró efecto del sexo sobre esta variable, el $\mathrm{pH}$ medido a las $24 \mathrm{~h}$ post morten, para hembras y machos, fue de 5.69 y 5.62; estos valores son muy parecidos a los reportados en este trabajo que fueron de 5.63 y 5.74 para hembras y machos. Bianchi, 2006 reportó valores de 5.6 y 5.7 para hembras y machos. Los siguientes autores reportaron valores parecidos (Fernandes et al., 2013; Marichal et al., 2003; Žgur et al., 2003).

\section{CONCLUSIÓN}

No se encontró efecto de la edad ni del sexo sobre el color y pH de la carne; sin embargo, la hora de muestreo si afectó las variables anteriores.

\section{LITERATURA CITADA}

AMS. 2012. Guidelines for Meat Color Evaluation. Proceeding of the Reciprocal Meat Conference. 44:3-17. w.w.w.meatscience.org. https://meatscience.org/docs/defaultsource/publications-resources/hottopics/2012_12_meat_clr_guide.pdf?sfvrsn=d818b8b3_0

ALBERTI P, Ripoll G. 2010. Los pigmentos de la carne y factores que afectan su color. En: Introducción a la Ciencia de la Carne. Ed. Hemisferio Sur, Buenos Aires Argentina. ISBN: 97-9974-674-202.

https://calidadcarnecita.wordpress.com/2012/06/28/introduccion-a-la-ciencia-de-lacarne/

BIANCHI G. 2006. Alternativas tecnológicas para la producción de carne ovina de calidad en sistemas pastoriles. Ed. Hemisferio Sur, Buenos Aires Argentina. ISBN: 97-9974-64549-3. https://isbn.cloud/9789974645493/alternativas-tecnologicas-para-la-produccionde-carne-ovina-de-calidad-en-sistemas-pastoriles/

CALNAN HB, Jacob RH, Pethick DW, Gardner GE. 2014. Factors affecting the color of lamb meat from Longissimum muscle during display. The influence of muscle weight and muscle oxidative capacity. Meat Science. 96: 1049-1057. ISSN: 2013.0.032. https://doi.org/10.1016/j.meatsci.2013.08.032 
CRAIGIE, CR, Lambe NR, Richardson RI, Haresign W, Maltin CA, Rehfeldt R, Morris ST, Bunger L. 2012. The effect of sex on some carcass and meat quality traits in Texel ewe and ram lambs. Animal Production Science. 52:601-607. ISSN: 136-0939. https://doi.org/10.1071/AN11282

FERNANDES JGA, Lobo RNB, Madruga MS, Lobo AMBO, Vieira LS, Faco O. 2013. Genotype effect on carcass and meat quality of lamb finished in irrigate pasture in the semiarid Northeaster Brazil. Arquivo Brasileiro de Medicina Veterinaria e Zootecnia. 65. ISSN: 0102-0935. https://doi.org/10.1590/S0102-09352013000400037

GUERRERO A, Velandia VM, Campo MM, Sañudo C. 2013. Some factors that affect ruminant meat quality: from the farm. to the fork. Review. Scientiarum. Animal Sciences $\begin{array}{lllll}\text { Maringá. } & 35 & (4): & 335-347 . & \text { ISSN: }\end{array}$ https://doi.org/10.4025/actascianimsci.v35i4.21756

MARICHAL A, Castro N, Capote J, Zamorano MJ, Argüello A. 2003. Effects of the live weights at slaughter $(6,10$ and $25 \mathrm{~kg})$ on kid carcass and meat quality. Livestock Production Science. 82: 247-256. ISSN: 0301-6226. https://doi.org/10.1016/S03016226(03)00113-1

MCGEEHIN D, Sheridan JJ, Butler F. 2001. Factors affecting the pH decline in lamb after slaughter. Meat Science. 58: 79-84. ISSN: 0309-1740. https://doi.org/10.1016/s03091740(00)00134-0

NEETHLING NE, Suman SP, Sigge GO, Hoffman LC, Hunt MC. 2017. Exogenous and Endogenous Factors Influencing Color of Fresh Meat from Ungulates. Meat and Muscle Biology. 1:253-275. ISSN: 2575-95X. https://doi.org/10.22175/mmb2017.06.0032d

PARTIDA de la PJA, Casaya RTA, Rubio LMS, Méndez MRD. 2015. Efecto del clorhidrato de zilpaterol sobre las características de la canal en cruzas terminales de corderos Kathadin. Veterinaria México. 2: 1-13. ISSN: 2448-6760. http://dx.doi.org/10.21753/vmoa.2.2.346

PASCUAL-ALONSO M, Aguayo-Ulloa L, Miranda de la Lama GC, Aliesta S, Olleta JL, Campo MM, Villarroel M, María GA. 2015. Effect of slaughter age on meat quality of Chamarito lambs. Animal Genetic Resources. 57:73-79. ISSN: 207-6336. https://doi.org/10.1017/S2078633615000156 
REZENDE MM, Ferrerira CR, Cenachi PDM, da Luz SS, Bonaguiro GS, Leme PR. 2017. Time on feedlot and sexual effects on animal performance and characteristics of lamb's meat. Acta Scientarum. Animal Sciences. 39:103-109. ISSN: 1806-2636. https://doi.org/10.4025/actascianimsci.v39i1.32749

RODRIGUES L, Gonçalvez CH, Lemos MBB, Furlan MM, Komiyama CM, Costa CM. 2011. Effect of genotype, finishing system, and sex on physiochemical characteristics of goat meat. Ciênc. Tecnol. Aliment., Campinas. 31:992-997. ISSN: 0101-2061. https://doi.org/10.1590/S0101-20612011000400027

SAGARPA. Secretaría de Agricultura, ganadería, desarrollo Rural, Pesca y Alimentación. 2017. La ovinocultura, una actividad muy arropadora. www.gob.mx. https://www.gob.mx/agricultura/es/articulos/la-ovinocultura-una-actividad-muyarropadora

SPSS. Statistical Package for the Social Sciences. 2017. Version 25. https://www.ibm.com/support/pages/downloading-ibm-spss-statistics-25

TARUMAN JA, Smulders JP, Gallo CB. 2018. Risk Factors for Bruises and High Muscle $\mathrm{pH}$ in Lambs Carcasses of Tierra del Fuego, Chilean Patagonia. Open Acces Library Journal. 5:1-11. ISSN: 2333-9705. https://doi.org/10.4236/oalib.1104291

YOUNG OA, Reid DH, Scales G.H. 1993. Effect of breed and ultimate pH on the odour and flavour of sheep meat. New Zealand Journal of Agricultural Research. (36): 363-370. ISSN: 002-233. https://doi.org/10.1080/00288233.1993.10417733

ŽGUR S, Cividine A, Kompas D, Birtiv D. 2003. The effect of Live Weight at Slaughter and sex on Lambs Carcass Traits and Meat Characteristics. Agriculturae. Conspectus Scientificus. 68:155-159. ISSN:1331-7776.

https://acs.agr.hr/acs/index.php/acs/article/view/222 